\title{
Planar Eleven Antenna as a Wideband MIMO Micro-base Station Antenna
}

\author{
Wenjie Yu, Jian Yang, Per-Simon Kildal \\ Dept. of Signals and Systems, Chalmers University of Technology, Gothenburg, Sweden \\ wenjiey@student.chalmers.se, jian.yang@chalmers.se, per-simon.kildal@chalmers.se
}

\begin{abstract}
A new low-profile planar eleven antenna is designed as a wideband MIMO antenna for micro-base stations in future wireless communication systems. The design criterion is to minimize both the reflection coefficient and the ratio of the required average received power over the threshold for $95 \%$ of the total probability of detection (PoD) in the Rich Isotropic Multipath (RIMP) and random Line-of-Sight (RLOS) scenarios of both one-bit stream and two-bit stream. The design is performed via optimization with a genetic algorithm.
\end{abstract}

Index Terms-MIMO antenna, Random Line-of-Sight, Rich Isotropic Multipath, probability of detection, genetic algorithm.

\section{INTRODUCTION}

Multi-input multi-output (MIMO) antennas operating over a wide frequency band are required in future wireless communication systems, such as in the $5 \mathrm{G}$ communication system.

The Eleven antenna is a dual-polarized ultra-wideband (UWB) antenna with a decade bandwidth. It has been used as a feed for reflector antennas [1] and demonstrated good performance in radio telescope applications [2]-[7]. In addition to the applications for reflector antennas, the multi-port Eleven antenna has been studied for use in such as monopulse tracking systems [8] and UWB communication systems as a MIMO antenna [9], [10].

In this paper, we propose a new low-profile flat MIMO Eleven antenna as shown in Fig. 1, including two branches located in two separate planes corresponding to two different polarizations orthogonal to each other, for the applications in future wireless communication systems. The flat Eleven MIMO antenna has a simple geometry and therefore a low manufacture cost. The design criteria is to minimize both the reflection coefficient and the ratio of the required average received power $P_{\mathrm{av}}$ over the threshold $P_{\mathrm{t}}$ with $95 \%$ of the total probability of detection (PoD). The operating scenario can be the Rich Isotropic Multipath (RIMP) and random Lineof-Sight (RLOS) for both one-bit stream and two-bit stream. The simulated reflection coefficient is below $-8 \mathrm{~dB}$, and the value of $P_{\mathrm{av}} / P_{\mathrm{t}}$ is below $4 \mathrm{~dB}$ at $95 \%$ of PoD in RLOS for one bitstream by zero forcing (ZF) algorithm. This antenna can work as micro-base station in $5 \mathrm{G}$ communication systems.

\section{THEORY AND FIGURE OF MERIT}

\section{A. Background of OTA}

Over-the-air (OTA) tests are widely applied to predict the reliability, safety and performance capabilities, etc. of real-

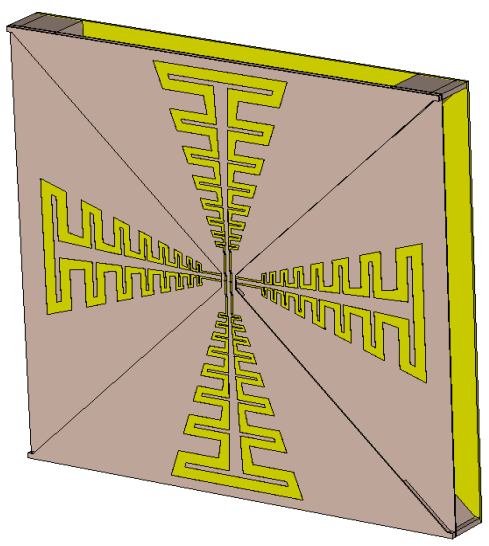

Fig. 1: CST model of the present flat MIMO Eleven antenna with a size of $337 \times 337 \times 37 \mathrm{~mm}^{3}$.

world wireless devices in modern communication systems.

To emulate the real-life environment, a new systematic characterization approach is proposed in [11] for OTA measurement evaluation of wireless devices in RIMP and RLOS, with a real-life OTA hypothesis which states that if a wireless device works well in both RIMP and random-LOS, it will also work well in real-life environment. The RLOS and RIMP environments are linked to real-life environments by the hypothesis and a new method to optimize the wireless network taking into account the user statistics is proposed in [12]. The RLOS environment can be analyzed with the ViRMlab, a computer program investigating performance of wireless terminals in multipath with many arbitrary incident waves [13] based on standard receive antenna equivalent circuits [14]. The way of characterizing the micro-BTS in terms of PoD in both RIMP and RLOS is proposed in [15].

\section{B. Theory}

1) RLOS: Line-of-Sight (LOS) propagation of an electromagnetic wave means the direct ray from the transmitter to the receiver is unobstructed, i.e., the transmission path is not established by or dependent upon reflection or diffraction. The anechoic chamber is traditionally used in antenna test for simulating the LOS environment. It was mainly used for antennas with directive beams of a known pointing direction. But antenna devices in modern communication systems are not limited to this type. It is often that antennas are not of a 
directive beam, but rather several arbitrarily oriented beams. Besides, the orientation of a wireless device, e.g. cell phone, is a kind of randomness. Then, when the distance between the base station and the user is short (the case for micro base station) or when the operating frequency is high at millimeter waves, the scenario of random line-of-sight may dominate. That's why RLOS [11] scenario, taking into account the user statistics, is studied in this paper.

2) RIMP: Multipath environment can be characterized by several independent incoming waves, whose amplitudes, phases, polarizations and the angles of arrival (AoA) are uncorrected to each other, at the receiving side [16]. The rich isotropic multipath environment is the hypothetical multipath environment defined as a reference, for the convenience of measurement, that assumes uniform distribution of AoA of the incoming waves within $4 \pi$ steradian and polarization balance, where the term 'rich' means the number of incoming waves is large, typically more than 100 [11].

3) PoD: The bit error rate (BER) is the percentage of bits that have errors relative to the total number of bits received in the receiver. BER is widely used as a criterion in wireless communication to measure the performance of the communication system. In advanced wireless systems, the bits are gathered in groups so that the bit group is less sensitive to errors than the single bit is. For this reason, the group error rate (GER) is introduced and an ideal error correction receiver is defined in [17], when a dynamic channel with time-varying fading is considered, as

$$
\operatorname{GRE}_{\mathrm{av}}\left(P_{\mathrm{av}}\right)=\operatorname{CDF}\left(P_{\mathrm{t}} / P_{\mathrm{av}}\right)
$$

where $P_{\mathrm{av}}$ is the average of received power and $P_{\mathrm{t}}$ is the threshold power level, under which the digital receiver with the error correction codes does not function correctly but above which it functions. The average throughput date rate $T_{P U T}$ is defined in [15] as

$$
T_{\mathrm{PUT}}\left(P_{\mathrm{av}}\right)=T_{\mathrm{PUT}, \max } \times\left[1-\operatorname{CDF}\left(P_{\mathrm{t}} / P_{\mathrm{av}}\right)\right]
$$

where $T_{\mathrm{PUT}}$, max is the maximum achievable throughput for the system and CDF is the cumulative distribution function of the channel power. The PoD as a function of the average received power is defined as

$$
\begin{aligned}
\operatorname{PoD}\left(P_{\mathrm{av}}\right) & =T_{\mathrm{PUT}}\left(P_{\mathrm{av}}\right) / T_{\mathrm{PUT}, \max } \\
& =1-\operatorname{CDF}\left(P_{\mathrm{t}} / P_{\mathrm{av}}\right)
\end{aligned}
$$

For the RLOS cases, the PoD is calculated within a certain solid angle area from the transmitting antenna (such as the antenna of a base station), illustrated as a red window on a sphere in Fig. 1. It is the intersection between the sphere and a right pyramid with an 4-sided base whose apex on the center of the sphere. In this work, The angle between the two pairs of the side faces facing each other in the pyramid is chosen as $120^{\circ}$.

Bitstream is defined as the independent informationcarrying modulated wave vectors coming in on each (or some)

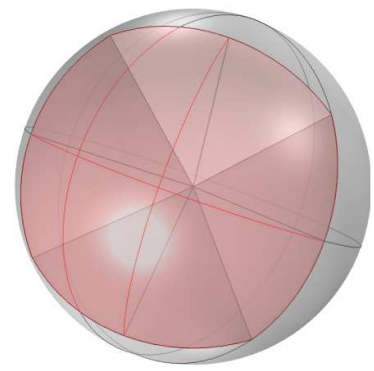

Fig. 2: Illustration of the solid angle area for PoD calculation in RLOS cases

TABLE I: Geometry Parameters of the Present Antenna for Optimization

\begin{tabular}{l|l}
\hline \hline Parameter & Definition \\
\hline$f_{\text {geo, max }}$ & highest geometry frequency \\
\hline$k$ & scaling factor \\
\hline$d_{a}$ & width of dipole \\
\hline$d_{c}$ & width of the gap in dipole \\
\hline$w_{a}$ & width of dipole arm \\
\hline$S$ & separation between transmission line \\
\hline$w_{t}$ & width of the transmission line \\
\hline \hline
\end{tabular}

of the ports of the transmitting antenna [15]. The PoD for a single bitstream case using Maximum-Ratio Combining (MRC), in which the output is a weighted sum of all branches [18] and for two bitstreams case using a simple Zero Forcing (ZF) algorithm[19] is of interest in this paper.

\section{Modeling AND Optimization}

\section{A. Layout}

The antenna is composed of two branches, i.e. the high branch and the low branch, in different planes separated by $3 \mathrm{~mm}$ vertically corresponding to two orthogonally polarized waves. The sizes of the eight dipole pairs are in geometric progression with a scaling factor $k$ between the previous pair and the next pair. The two parts in each branch are connected with a transmission line, in the middle of which the antenna is excited through coaxial cables. The total size of the antenna is $337 \times 337 \times 37 \mathrm{~mm}^{3}$ (see Fig. 1 and details in Fig. 3).

\section{B. Modeling}

The antenna is supposed to fulfill the requirement of wide bandwidth, e.g. over the frequency band from 1.6 to $2.8 \mathrm{GHz}$, at $95 \%$ PoD under the condition of both RLOS and RIMP and low reflection coefficient $\left(S_{11}\right)$. To fulfil the requirement on bandwidth, multiple dipoles scaled with a scaling factor $k$ are cascaded. The larger the number of the dipoles cascaded is, the wider the bandwidth over which the PoD is acceptable. It is observed that 8 dipoles are enough to achieve the bandwidth requirement. The other criteria, i.e. $S_{11}$ and PoD over the frequency band of interest, are dependent on all of the geometric parameters, i.e. $f_{\text {geo, } \max }, k, d_{a}, d_{c}, w_{a}, S$, and $w_{t}$ of the antenna, with the definition shown in Table I.

To find the initial values of the geometric parameters for the optimization, we tune all parameters one by one while keeping 


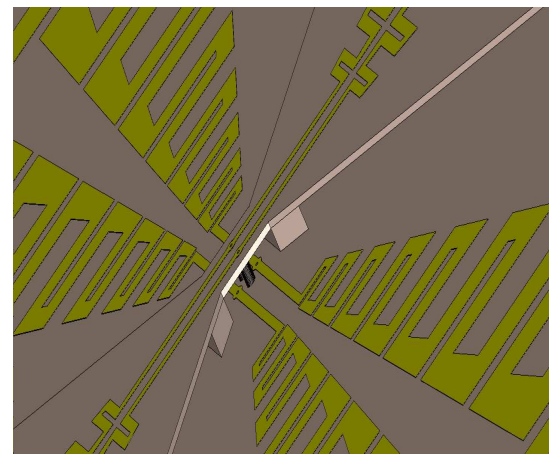

(a) Transmission line part

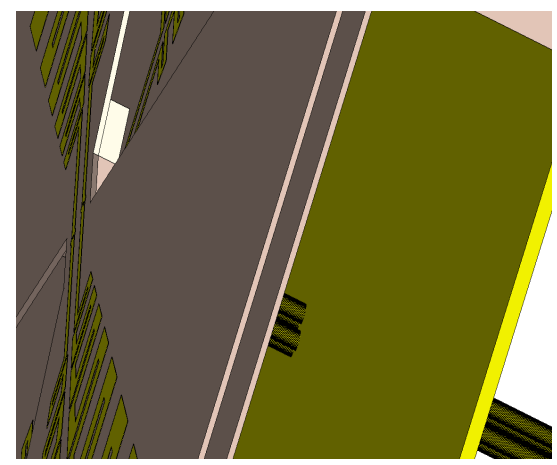

(b) Side view

Fig. 3: Detail of the CST model of the flat MIMO Eleven antenna.

others fixed. The function of every parameter i.e. how it will affect the $S_{11}$ can be observed through this process. Then, a bunch of parameters as the initial values and the parameter scanning range have been determined.

\section{Optimization}

The antenna is optimized employing the so-called Genetic Algorithm [20]. The geometric parameters $f_{\text {geo, } \max }, k, d_{a}, d_{c}$, $w_{a}, S$, and $w_{t}$, which determine the shape and total size of the antenna, will influence both the $S_{11}$ and the PoD, and thus are treated as genes. Four hundred samples whose genes are randomly generated and uniformly distributed within the range obtained through parameter scanning previously are evaluated by using CST in the first generation.

The 400 samples are ramked according to the value of their maximum $S_{11}$ over the frequency band of 1.6 to $2.8 \mathrm{GHz}$. The PoD of all the samples is also evaluated via ViRM-Lab by the simulated far-field function. Fifteen samples of the four hundred with the lowest maximum $S_{11}$ and acceptable $\mathrm{PoD}$ are selected as elites and given the chance to mate each other pairwise. Each pair will generate 2 children. Then, the Roluttee-Wheel selection rule is used to pick other fifteen samples in the rest 385 samples to generate offsprings in the same way as the elites. The optimization runs for 3 generations and the result $\left(S_{11}\right)$ converges.

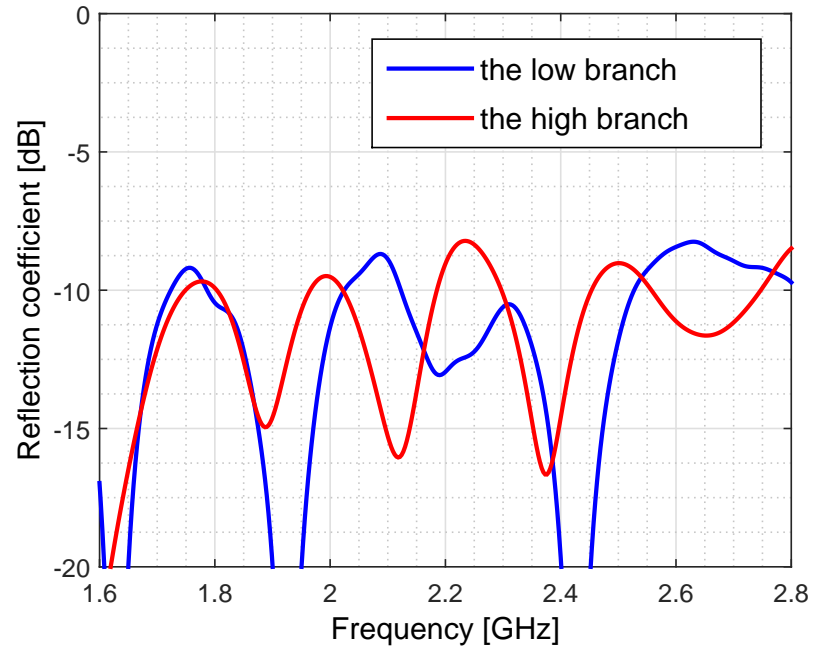

Fig. 4: CST simulated reflection coefficient of the present antenna after optimization

\section{Simulation Results}

The $S_{11}$ and the probability of detection under both the RIMP situation and RLOS situation of the antenna are simulated.

The maximum $S_{11}$ over the band is minimized using our genetic algorithm over generations. For the high branch and the low branch of the antenna petals over the frequency of interest the maximum $S_{11}$ is $-8.22 \mathrm{~dB}$ and $-8.25 \mathrm{~dB}$, respectively, as shown in Fig. 4.

The required $P_{\mathrm{av}} / P_{\mathrm{t}}$ to reach $95 \%$ probability of detection under RLOS and RIMP for one bitstream using MRC and for two bitstreams using ZF are plotted in Fig. 5.

It can be seen from the figure the followings. i) In one bitstream MRC under RIMP scenario, the average received power $P_{\mathrm{av}}$ at the terminals (mobile phones or other wireless devices) should be $9 \mathrm{~dB}$ higher than its throughput threshold power level $P_{\mathrm{t}}$ (which is normally between -80 and -90 $\mathrm{dBm}$ ) in order to guarantee $95 \%$ of wireless devices function correctly, while in two bitstream MRC under RIMP scenario, $P_{\text {av }}$ should be $17 \mathrm{~dB}$ higher than $P_{\mathrm{t}}$ since each branch of the Eleven antenna has its own channel. ii) For RLOS case, the required average received power $P_{\mathrm{av}}$ in the area shown in Fig. 2 is only about $3 \mathrm{~dB}$ higher than $P_{\mathrm{t}}$ in one bitstream ZF, and 12 $\mathrm{dB}$ higher than $P_{\mathrm{t}}$ in two bitstream ZF. Therefore, the present MIMO eleven antenna works very well in one bitstream ZF under RLOS scenario over the frequency band with nearly no variations.

\section{CONCLUSION}

A novel planar type Eleven Antenna for micro-base station working on $1.6 \mathrm{GHz}$ to $2.8 \mathrm{GHz}$ is designed. The $S_{11}$ and probability of detection under RLOS and RIMP for one bitstream using MRC and two bitstreams using ZF over frequency of $1.6-2.8 \mathrm{GHz}$ are simulated and optimized. 


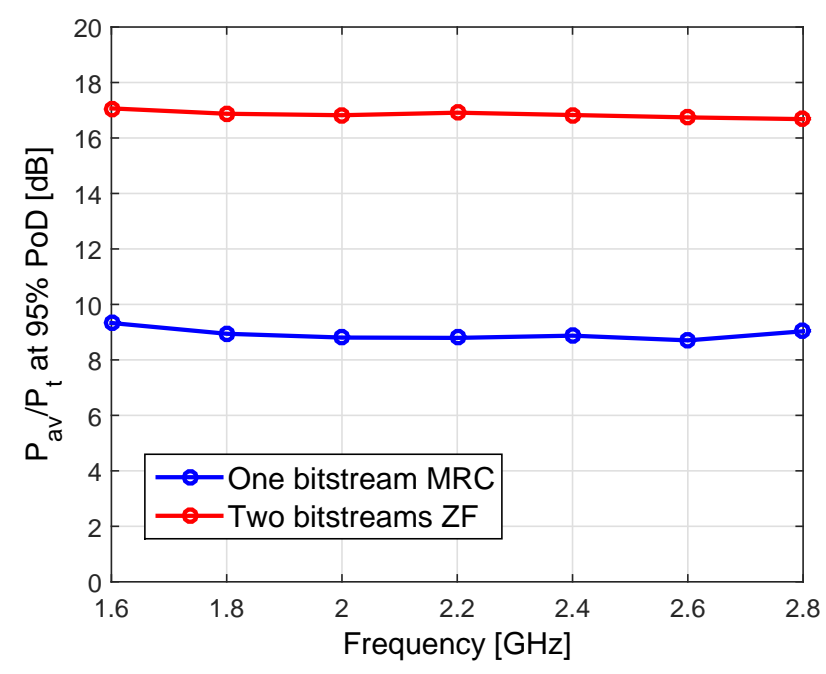

(a) Under RIMP situation

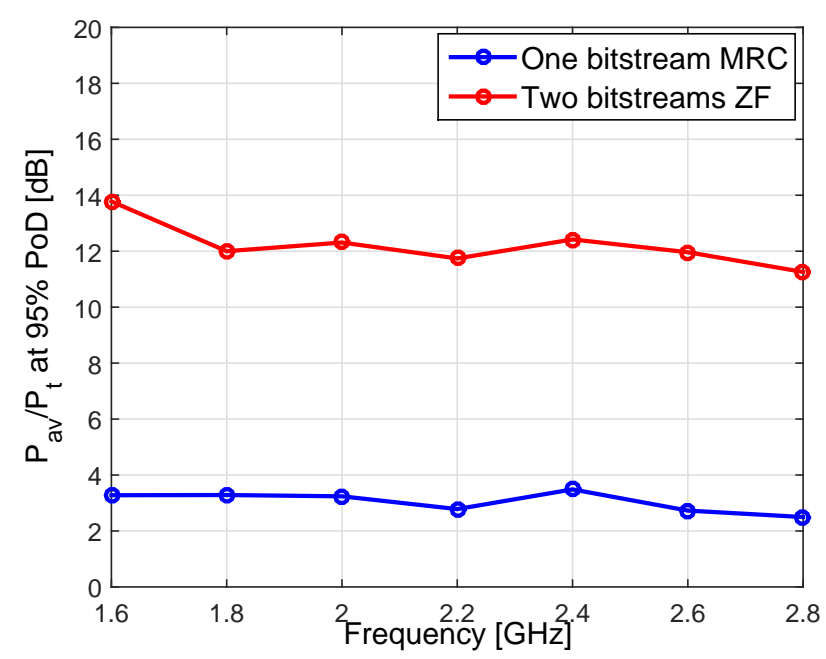

(b) Under RLOS situation

Fig. 5: Simulated required $P_{\mathrm{av}} / P_{\mathrm{t}}$ in order to reach $95 \% \mathrm{PoD}$ by using ViRM-Lab

\section{ACKNOWLEDGMENT}

Wenjie Yu would like to thank Dr. Ulf Carlberg from Qualcomm for providing the 'ViRM-Lab' code for Figure 5(a) and Figure 5(b) and Junfei Tang from Chalmers University of Technology for making Figure 2.

\section{REFERENCES}

[1] P.-S. Kildal, R. Olsson, and J. Yang, "Development of three models of the eleven antenna: A new decade bandwidth high performance feed for reflectors," in Antennas and Propagation, 2006. EuCAP 2006. First European Conference on, Nov 2006, pp. 1-6.

[2] R. Olsson, P.-S. Kildal, and S. Weinreb, "The eleven antenna: a compact low-profile decade bandwidth dual polarized feed for reflector antennas," Antennas and Propagation, IEEE Transactions on, vol. 54, no. 2, pp. 368-375, Feb 2006
[3] J. Yang, X. Chen, N. Wadefalk, and P.-S. Kildal, "Design and realization of a linearly polarized eleven feed for 1-10 ghz," Antennas and Wireless Propagation Letters, IEEE, vol. 8, pp. 64-68, 2009.

[4] J. Yang, D. Nyberg, and J. Yin, "Impedance matrix of a folded dipole pair under eleven configuration," Microwaves, Antennas Propagation, IET, vol. 4, no. 6, pp. 697-703, June 2010.

[5] J. Yang, "On conditions for constant radiation characteristics for logperiodic array antennas," Antennas and Propagation, IEEE Transactions on, vol. 58, no. 5, pp. 1521-1526, May 2010.

[6] J. Yang and P.-S. Kildal, "Optimization of reflection coefficient of large log-periodic array by computing only a small part of it," Antennas and Propagation, IEEE Transactions on, vol. 59, no. 6, pp. 1790-1797, June 2011.

[7] J. Yang, M. Pantaleev, P.-S. Kildal, B. Klein, Y. Karandikar, L. Helldner, N. Wadefalk, and C. Beaudoin, "Cryogenic 2-13 ghz eleven feed for reflector antennas in future wideband radio telescopes," Antennas and Propagation, IEEE Transactions on, vol. 59, no. 6, pp. 1918-1934, June 2011.

[8] J. Yin, J. Aas, J. Yang, and P. Kildal, "Monopulse tracking performance of multi-port eleven antenna for use in satellite communications terminals," in Antennas and Propagation, 2007. EuCAP 2007. The Second European Conference on, Nov 2007, pp. 1-4.

[9] J. Yang, S. Pivnenko, T. Laitinen, J. Carlsson, and X. Chen, "Measurements of diversity gain and radiation efficiency of the eleven antenna by using different measurement techniques," in Antennas and Propagation (EuCAP), 2010 Proceedings of the Fourth European Conference on, April 2010, pp. 1-5.

[10] X. Chen, P.-S. Kildal, J. Carlsson, and J. Yang, "Comparison of ergodic capacities from wideband mimo antenna measurements in reverberation chamber and anechoic chamber," Antennas and Wireless Propagation Letters, IEEE, vol. 10, pp. 446-449, 2011.

[11] P.-S. Kildal and J. Carlsson, "New approach to ota testing: Rimp and pure-los as extreme environments \& a hypothesis [elektronisk resurs]," 7th European Conference on Antennas and Propagation, EuCAP 2013, Gothenburg, Sweden, 8-12 April 2013, vol. s. 315-318, 2013.

[12] P.-S. Kildal, "Rethinking the wireless channel for ota testing and network optimization by including user statistics: Rimp, pure-los, throughput and detection probability," in 2013 Proceedings of The International Symposium on Antennas and Propagation (ISAP), Vols 1 and 2.

[13] U. Carlberg, J. Carlsson, A. Hussain, and P.-S. Kildal, "Ray based multipath simulation tool for studying convergence and estimating ergodic capacity and diversity gain for antennas with given far-field functions," in ICECom, 2010 Conference Proceedings, Sept 2010, pp. $1-4$.

[14] P.-S. Kildal, "Equivalent circuits of receive antennas in signal processing arrays," Microwave and Optical Technology Letters, vol. 21, pp. 244246, 1999.

[15] P.-S. Kildal, X. Chen, M. Gustafsson, and Z. Shen, "Mimo characterization on system level of $5 \mathrm{~g}$ microbase stations subject to randomness in los," Access, IEEE, vol. 2, pp. 1064-1077, 2014.

[16] P.-S. Kildal, Foundations of Antenna Engineering: A Unified Approach for Line-Of-Sight and Multipath. Kildal Antenn AB, 2015.

[17] P.-S. Kildal, A. Hussain, X. Chen, C. Orlenius, A. Skarbratt, J. Asberg, T. Svensson, and T. Eriksson, "Threshold receiver model for throughput of wireless devices with mimo and frequency diversity measured in reverberation chamber," Antennas and Wireless Propagation Letters, IEEE, vol. 10, pp. 1201-1204, 2011.

[18] A. Goldsmith, Wireless Communications. New York, NY, USA Cambridge University Press, 2005.

[19] X. Chen, P.-S. Kildal, and M. Gustafsson, "Characterization of implemented algorithm for mimo spatial multiplexing in reverberation chamber," Antennas and Propagation, IEEE Transactions on, vol. 61, no. 8, pp. 4400-4404, Aug 2013.

[20] Y. Rahmat-Samii and E. Michielssen, Eds., Electromagnetic Optimization by Genetic Algorithms, 1st ed. New York, NY, USA: John Wiley \& Sons, Inc., 1999. 